The University of San Francisco

USF Scholarship: a digital repository @ Gleeson Library |

Geschke Center

2013

\title{
Characterization of the Supersonic Flowing Microwave Discharge Using Two Dimensional Plasma Tomography
}

Milka Nikolic

University of San Francisco, mnikolic@usfca.edu

A. Samolov

F. Čučkov

S. Popović

L. Vušković

See next page for additional authors

Follow this and additional works at: http://repository.usfca.edu/phys

Part of the Physics Commons

\section{Recommended Citation}

Nikolić, M., Samolov, A., Čučkov, F., Popović, S., Vušković, L., Godunov, A. Characterization of the supersonic flowing microwave discharge using two dimensional plasma tomography (2013) Journal of Applied Physics, 113 (10), art. no. 103301. http://dx.doi.org/ $10.1063 / 1.4793494$ 
Authors

Milka Nikolic, A. Samolov, F. Čučkov, S. Popović, L. Vušković, and A. Godunov 
Characterization of the supersonic flowing microwave discharge using two dimensional plasma tomography

M. Nikolić, A. Samolov, F. Čučkov, S. Popović, L. Vušković, and A. Godunov

Citation: Journal of Applied Physics 113, 103301 (2013); doi: 10.1063/1.4793494

View online: http://dx.doi.org/10.1063/1.4793494

View Table of Contents: http://aip.scitation.org/toc/jap/113/10

Published by the American Institute of Physics

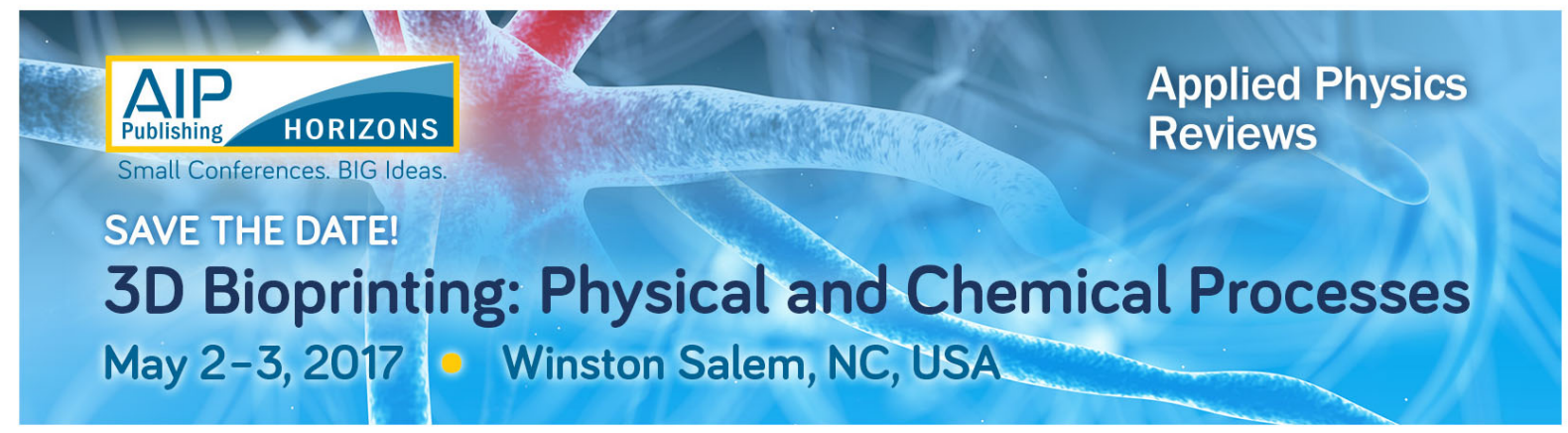




\title{
Characterization of the supersonic flowing microwave discharge using two dimensional plasma tomography
}

\author{
M. Nikolić, ${ }^{1}$ A. Samolov, ${ }^{1}$ F. Čučkov, ${ }^{2}$ S. Popović, ${ }^{1}$ L. Vušković, ${ }^{1}$ and A. Godunov ${ }^{1}$ \\ ${ }^{1}$ Department of Physics, Center for Accelerator Science, Old Dominion University, Norfolk, \\ Virginia 23529, USA \\ ${ }^{2}$ Department of Electrical and Computer Engineering, Old Dominion University, Norfolk, \\ Virginia 23529, USA
}

(Received 4 December 2012; accepted 11 February 2013; published online 11 March 2013)

\begin{abstract}
A tomographic numerical method based on the two-dimensional Radon formula for a cylindrical cavity has been employed for obtaining spatial distributions of the argon excited levels. The spectroscopy measurements were taken at different positions and directions to observe populations of excited species in the plasmoid region and the corresponding excitation temperatures. Excited argon states are concentrated near the tube walls, thus, confirming the assumption that the post discharge plasma is dominantly sustained by travelling surface wave. An automated optical measurement system has been developed for reconstruction of local plasma parameters of the plasmoid structure formed in an argon supersonic flowing microwave discharge. The system carries out angle and distance measurements using a rotating, flat mirror, as well as two high precision stepper motors operated by a microcontroller-based system and several sensors for precise feedback control. () 2013 American Institute of Physics. [http://dx.doi.org/10.1063/1.4793494]
\end{abstract}

\section{INTRODUCTION}

There is a continuing interest in microwave discharges due to the wide variety of possible applications, such as material processing, surface modification, environmental protection, and aerodynamics of ionized gases. In order to characterize these discharges without perturbing them, it is possible to apply nonintrusive optical measurements. Another possibility would be to develop a numerical model of the plasma, based on the given experiment setup. ${ }^{1,2}$ The experimental, nonintrusive, and optical techniques available for plasma characterization allow us to measure only integrated effects of collective plasma behaviour. In order to look into the internal dynamics of the discharge, the integrated data need to be transformed into the spatial population distributions. A commonly used method for reconstructing an inner plasma structure is the Abel inversion, which requires plasma to be axially symmetric. ${ }^{3}$ As a rule, most plasmas of interest are nonstationary, inhomogeneous, and with strong radial and axial asymmetry. It follows that the discharge should be observed from at least two different angles. Plasma tomography technique based on an inverse Radon transform is an excellent way to acquire insight into the internal plasma structure. ${ }^{4}$ In previous years, extensive studies were conducted on the simplified case, where only two mutually perpendicular directions were used for tomography measurements. ${ }^{5,6}$ This approximation includes only the first two terms in the Fourier transform of the Radon integral, making it difficult to depict the entire angular distribution of plasma parameters. The Radon technique was described in literature using a four-axis industrial robot to study RF capacitively and inductively coupled plasmas. ${ }^{7}$

In this paper, we study the applicability of the general form of the Radon transform on the supersonic flowing microwave discharge maintained in a cylindrical quartz cavity. For that purpose, an automated measurement system (AMS) has been built in order to obtain a sliced two-dimensional (2D) image of population densities of different argon excited states. In particular, we focused on $\operatorname{Ar~I~}\left[3 s^{2} 3 p^{5}\left({ }^{2} P_{1 / 2}^{0}\right) 4 p, J=2 \rightarrow\right.$ $\left.3 s^{2} 3 p^{5}\left({ }^{2} P_{3 / 2}^{0}\right) 4 s, J=2\right]$ and $\left[3 s^{2} 3 p^{5}\left({ }^{2} P_{1 / 2}^{0}\right) 4 p, J=1 \rightarrow 3 s^{2}\right.$ $\left.3 p^{5}\left({ }^{2} P_{3 / 2}^{0}\right) 4 s, J=2\right]$ transitions at $706.72 \mathrm{~nm}$ and $714.70 \mathrm{~nm}$, respectively. The AMS carries out measurements of specific spectral line intensities at a number of positions at angles ranging from $0^{\circ}$ to $180^{\circ}$.

Our goal is to fully characterize the spatial plasma distribution by determining distributions of argon excited levels. By assuming that the excited states were in partial local thermodynamic equilibrium (PLTE), we estimated the 2D distribution of the electron excitation temperatures inside the MW discharge. We present a detailed description of the experimental setup, a cavity MW discharge in the supersonic flow. Further, we review the basics of the applied method. Finally, experimental results were presented and discussed.

\section{EXPERIMENTAL SETUP}

The experiment was conducted in a supersonic flowing tube in combination with microwave cavity discharge, shown in Fig. 1. A commercial microwave generator, operating in the S-band at $2.45 \mathrm{GHz}$, was used to sustain a cylindrical cavity discharge at power density of $4 \mathrm{~W} / \mathrm{cm}^{3}$. The working pressure in the evacuated quartz tube was 2.4 Torr. A Mach 2, a cylindrical convergent-divergent De Laval nozzle, was used to sustain the supersonic flowing discharge downstream the microwave cavity. The measurements were performed in pure argon that was fed into the stagnation chamber through a gas manifold. Gas flow was established by a roots blower (Pfeiffer Okta 500 A), which was supported by two roughing pumps (Varian SD-700). 


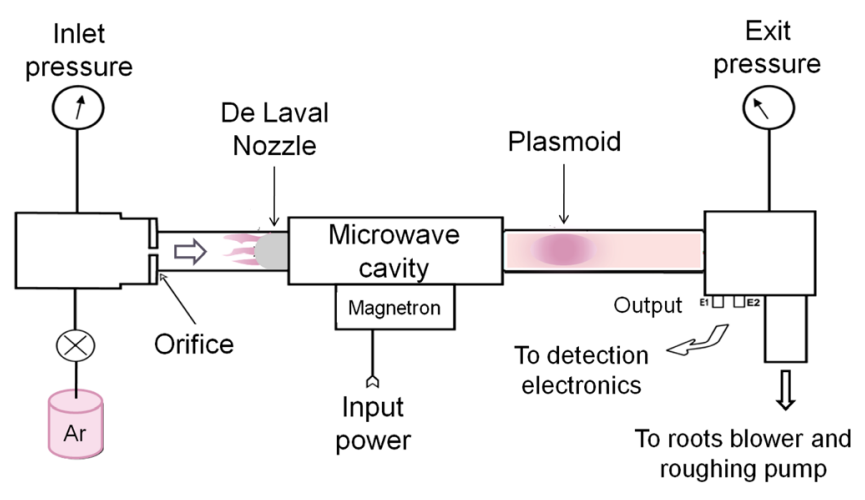

FIG. 1. Scheme of the supersonic flowing microwave discharge.

We employed optical emission spectroscopy as a diagnostic tool to observe the spectra of the Ar excited states. Emission spectra were recorded using two different detection systems: an imaging spectrograph (Acton SpectraPro) connected to the charged-coupled device (CCD) camera (Apogee, Hamamatsu, Back-Illum) and spectrometer (Ocean Optics HR4000CG) with the in-built CCD array detector (Toshiba). All spectral measurements were performed side-on with respect to the direction of the discharge flow.

In order to increase the precision of experimental measurements, we have built the AMS. It consists of a flat mirror and a set of feedback sensors connected to two high-precision stepper motors driven by a microcontroller-based system, as shown in Fig. 2. The precision of the selected bi-polar stepper motors, with up to 400 steps per revolution, allowed for a sub-millimeter and sub-degree control of the mirror's orientation with respect to the plasmoid. Each motor was connected to a rotational encoder wheel, which provided rotationalposition feedback to the controller. Additionally, the distance of the mirror from the quartz tube was measured by a linear sliding potentiometer, and the absolute angle of the mirror was measured by a triple axis accelerometer (Analog Devices ADXL335). Once the motors and sensors were connected to the rotational and translational knobs of the mirror platform, they were calibrated by visual inspection using a laser setup. The motors were driven by a Microstepping Driver with Translator (Allegro MicroSystems A3967SLB), connected to an 8-bit microcontroller (Atmel AVR ATmega328).

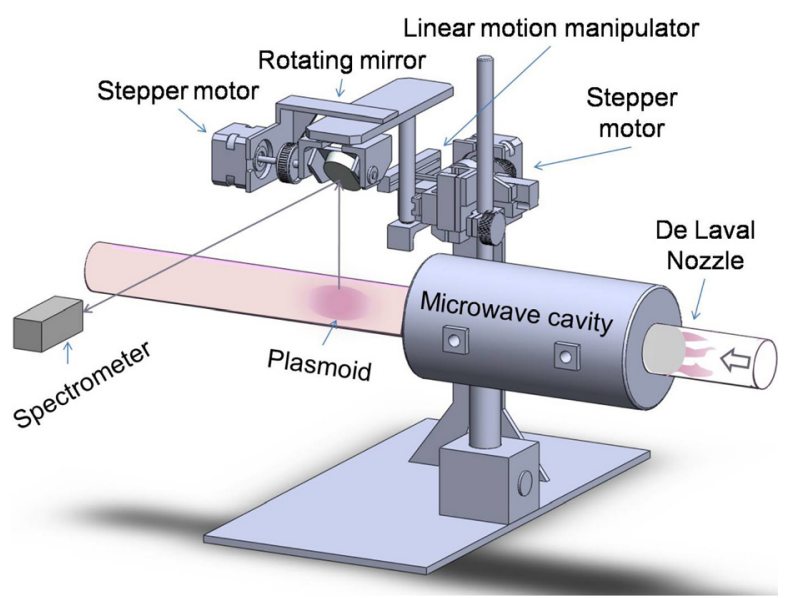

FIG. 2. Scheme of automated measurement system.
The microcontroller was connected, as a master, to a host computer via a USB serially emulated interface. The host computer was programmed to manage the microcontroller, motors, and sensors and to automate the measuring tasks.

By changing the angle and the position of the mirror, we were able to reflect the emitted light perpendicular to the spectrometer, which allowed us to keep the detecting system at a fixed position. The measurements were taken at three different positions from the cylindrical cavity, which correspond to both ends and the mid section of the plasmoid. This experimental setup allowed us to record projections under 21 angles in the range from $48^{\circ}$ to $168^{\circ}$ and 17 projections for each angle with the sampling rate of $0.2 \mathrm{~cm}$ across the diameter of the quartz tube.

The observed spectra were calibrated using a Newport/ Oriel absolute black body irradiance source. We evaluated the calibration graphs of spectral irradiance per count versus wavelength for wavelengths between 200 and $1100 \mathrm{~nm}$. Using these graphs, we determined the population of particular Ar excited state transitions. The black body irradiance calibration was obtained with the AMS included in the experimental setup, making redundant the need to measure the mirror reflectivity separately.

By using a standard back reflector procedure, we eliminated the possibility that the optical thickness of measured spectral line intensity was interfering with our measurement. Both spectral line intensities were higher by a factor of two in comparison to the direct plasma observation. Therefore, the radiation trapping along the observation path could be neglected.

\section{PLASMA TOMOGRAPHY}

A scheme of the experimental setup for the plasma tomography is shown in Fig. 3. We measured the spectral line intensities, $I(p, \theta)$, employing optical emission spectroscopy along a system of direct rays in a direction defined by the projection angle $\theta$ and distance $p$ from the origin. These intensities were used in the Radon formula, ${ }^{4}$ to provide information about the spatial population distribution, $\varepsilon(\mathrm{x}, \mathrm{y})$. We start by introducing the Radon transform integral

$$
I(p, \theta)=\Re[\varepsilon(x, y)]=\int_{L} \varepsilon(x, y) \mathrm{d} s,
$$

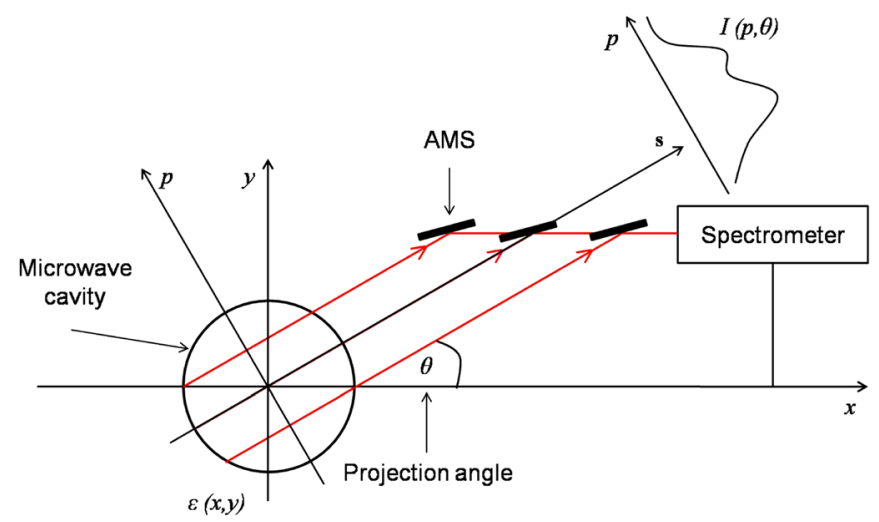

FIG. 3. Scheme of the experimental setup for our plasma tomography experiment. 
where $L$ is the line of integration, $d s$ is the increment of the length along that line, and

$$
\begin{gathered}
s=x \cos \theta+y \sin \theta, \\
p=-x \sin \theta+y \cos \theta .
\end{gathered}
$$

In order to retrieve the spatial population distribution from the measured spectral intensities, we need to invert the Radon integral

$$
\varepsilon(x, y)=\Re^{-1}[I(p, \theta)] .
$$

The complete derivation of the Radon inverted integral, using the two-dimensional Fourier transform in frequency domain, of unknown spatial distribution function was done somewhere else. ${ }^{8}$ By applying the Fourier slice theorem, measured spectral line intensities can be expressed as a set of projections $I_{\theta}(p)$ for different angles $\theta$, and the equation for the unknown spatial distribution function is

$$
\varepsilon(x, y)=\int_{0}^{\pi} \mathrm{d} \theta \int_{-\infty}^{\infty} \mathrm{d} \nu|\nu| F\left(I_{\theta}(p)\right) \exp \left(i 2 \pi \nu p_{0}\right) .
$$

Equation (5) can be rewritten as ${ }^{9}$

$$
\varepsilon(x, y)=\int_{0}^{\pi} Q_{\theta}(-x \sin \theta+y \cos \theta) \mathrm{d} \theta,
$$

where

$$
Q_{\theta}(p)=\int_{-\infty}^{\infty} \mathrm{d} \nu|\nu| F\left(I_{\theta}(p)\right) \exp \left(i 2 \pi \nu p_{0}\right) .
$$

In principle, the integration in filtered projection given by Eq. (6) has to be carried over all frequencies. However, in practice, the energy contained in the Fourier transform components above a certain frequency is negligible, and we may consider the projections bandlimited. When the highest frequency in the projections is finite, Eq. (7) becomes

$$
Q_{\theta}(p)=\int_{-\infty}^{\infty} F\left(I_{\theta}(p)\right) H(\nu) \exp \left(i 2 \pi \nu p_{0}\right) \mathrm{d} \nu,
$$

where $H(\nu)$ is a high frequency noise filter.

By applying the inverse Fourier transformation and the convolution theorem for each projection $I_{\theta}(p)$, we obtain

$$
Q_{\theta}(p)=\int_{-\infty}^{\infty} I_{\theta}\left(p^{\prime}\right) h\left(p-p^{\prime}\right) d p^{\prime} .
$$

Experimentally obtained line intensities are presented as a discrete set of projections, measured with the spatial sampling interval $\tau$. Thus, $p=n \tau$ where $n$ is an integer. If we assume that each projection $I_{\theta}(k \tau)$ is zero outside the index range $k=0,1, \ldots, K-1$, where $K$ is the number of angles at which the projections are sampled, we may express the filtered projection as

$$
\begin{aligned}
Q_{\theta}(n \tau) & =\tau \sum_{k=0}^{K-1} h(n \tau-k \tau) I_{\theta}(k \tau) \\
n & =0,1, \ldots, K-1
\end{aligned}
$$

Finally, the reconstructed function $\epsilon(x, y)$ may be obtained by the discrete approximation of Eq. (6)

$$
\varepsilon(x, y)=\frac{\pi}{K} \sum_{i=1}^{K} Q_{\theta_{i}}\left(-x \sin \theta_{i}+y \cos \theta_{i}\right) .
$$

This means that each filtered projection has to be backprojected.

In this work, we used the Shepp-Logan filter, ${ }^{10}$ shown in Fig. 4(a), commonly used filter in literature

$$
h(n \tau)= \begin{cases}\frac{2}{\pi^{2} \tau^{2}}, & \mathrm{n}=0 \\ -\frac{2}{n^{2} \pi^{2}\left(4 \nu^{2}-1\right)}, & \mathrm{n} \neq 0 .\end{cases}
$$

\section{RESULTS AND DISCUSSION}

In the afterglow of the MW supersonic flow region, we observed a plasmoid-like formation as a secondary downstream phenomenon coupled to the microwave cavity. The plasmoid appears to be sustained by a low power surface wave, which propagates along its surface and the surface of the quartz tube. The full interpretation of the plasmoid discharge requires knowledge of all effects causing its formation. For that reason, we calculated spatial population distributions and corresponding excitation temperatures of two neutral Ar excited states $\left[3 s^{2} 3 p^{5}\left({ }^{2} P_{1 / 2}^{0}\right) 4 p, J=2\right]$ and $\left[3 s^{2} 3 p^{5}\left({ }^{2} P_{1 / 2}^{0}\right) 4 p, J=1\right]$.

\section{A. Population densities}

Two spectral lines,

- $\left[3 s^{2} 3 p^{5}\left({ }^{2} P_{1 / 2}^{0}\right) 4 p, J=2 \rightarrow 3 s^{2} 3 p^{5}\left({ }^{2} P_{3 / 2}^{0}\right) 4 s, \quad J=2\right]$ at $706.72 \mathrm{~nm}$,

- $\left[3 s^{2} 3 p^{5}\left({ }^{2} P_{1 / 2}^{0}\right) 4 p, J=1 \rightarrow 3 s^{2} 3 p^{5}\left({ }^{2} P_{3 / 2}^{0}\right) 4 s, \quad J=2\right]$ at $714.70 \mathrm{~nm}$,

were used for determining the population of Ar excited states. In the case of the optically thin plasma, the spectral line intensity is proportional to the number of atoms

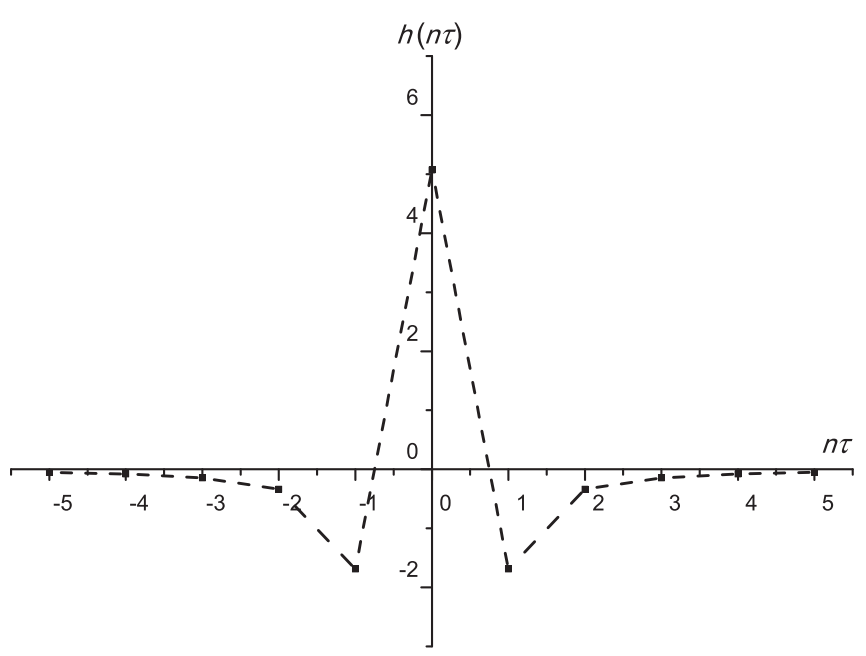

FIG. 4. Shepp-Logan filter in spatial domain. 
a) $3 \mathrm{~cm}$ from the cavity

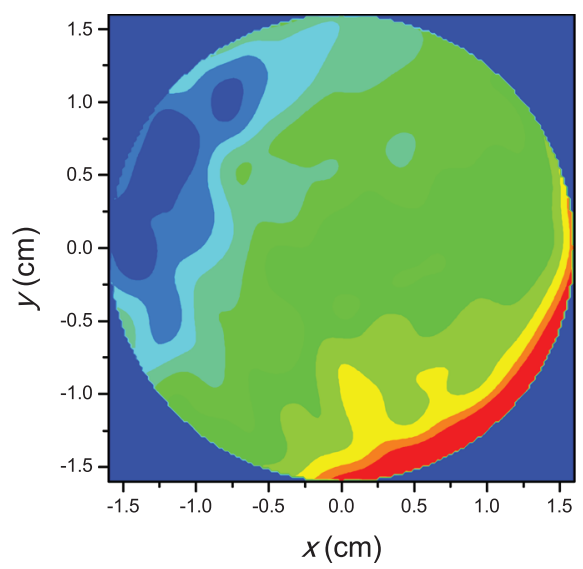

b) $4.5 \mathrm{~cm}$ from the cavity

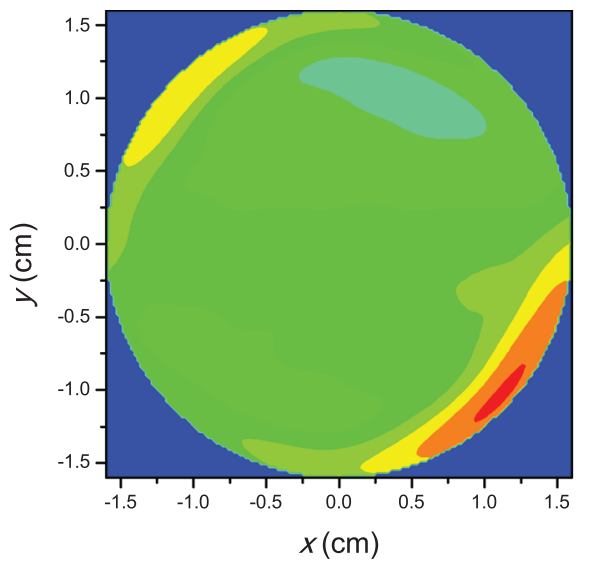

c) $8 \mathrm{~cm}$ from the cavity

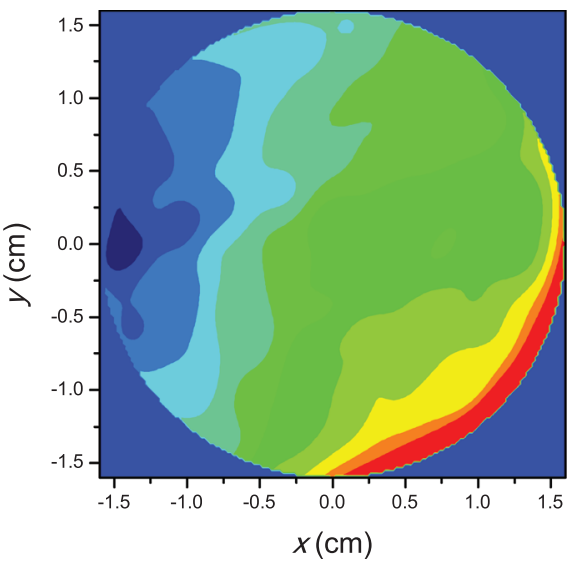

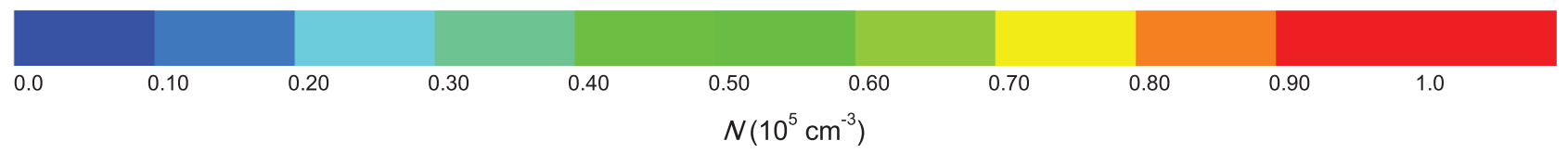

FIG. 5. Spatial distribution of the Ar I $\left[3 s^{2} 3 p^{5}\left({ }^{2} P_{1 / 2}^{0}\right) 4 p, J=2\right]$ population density using filtered back projection with Shepp-Logan filter obtained at distance (a) $3 \mathrm{~cm}$ from the cavity, (b) $4.5 \mathrm{~cm}$ from the cavity, and (c) $8 \mathrm{~cm}$ from the cavity.

populating the higher energy level. The population was evaluated at three different positions across plasmoid relative to the cavity. The first measurements were taken $3 \mathrm{~cm}$ from the cavity corresponding to the beginning of the plasmoid. The next position measured was at $4.5 \mathrm{~cm}$ from the cavity that corresponds to the middle range of the plasmoid. Finally, we observed the end of the plasmoid by taking measurements $8 \mathrm{~cm}$ from the cavity.

Figure 5 shows how the spatial distribution of Ar I excited states at $706.72 \mathrm{~nm}$ is changing along the plasmoid. It could be seen that the argon atoms are mainly populated close to the inner surface of the quartz tube. At distances $3 \mathrm{~cm}$ and $8 \mathrm{~cm}$ from the cavity (two ends of the plasmoid), argon atoms are concentrated close to one side of the quartz tube; while in the middle $(4.5 \mathrm{~cm}$ from the cavity), we observe almost symmetrical population distributions. The maximal value population density reaches at the surface of the tube is in the middle of the plasmoid and is $N=1.3 \times 10^{5} \mathrm{~cm}^{-3}$.

Similar results are observed for population distributions of Ar I excited states at $714.70 \mathrm{~nm}$, see Fig. 6. Argon atoms are distributed mostly at the surface of the tube with the maximal population density $N=1.6 \times 10^{5} \mathrm{~cm}^{-3}$.

\section{a) $3 \mathrm{~cm}$ from the cavity}

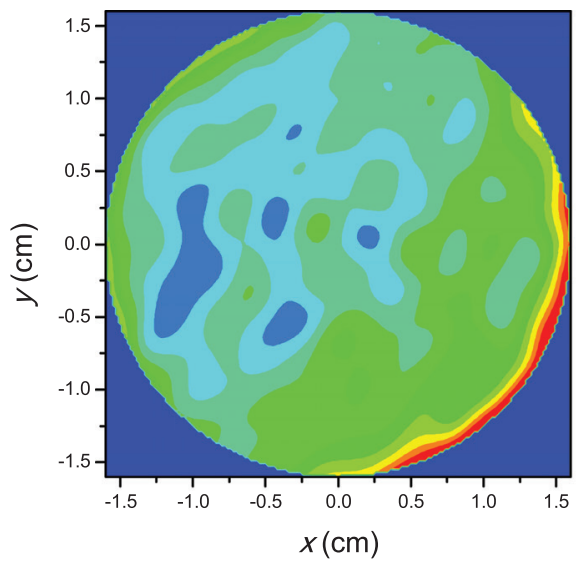

b) $4.5 \mathrm{~cm}$ from the cavity

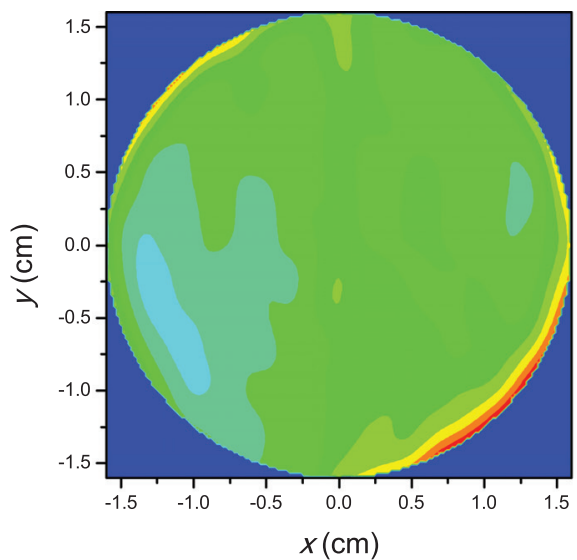

\section{c) $8 \mathrm{~cm}$ from the cavity}

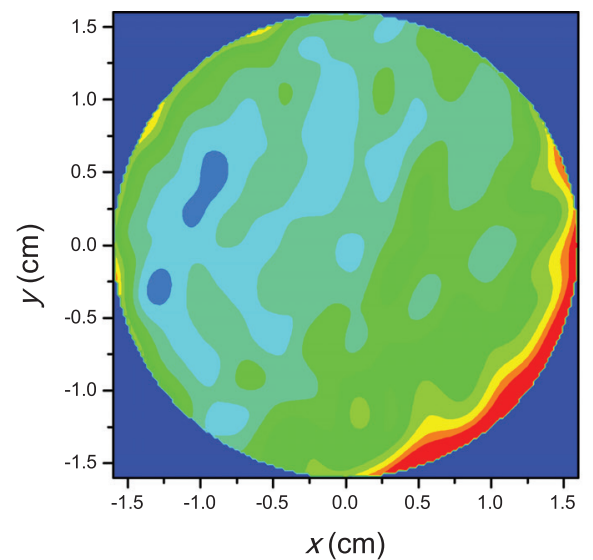

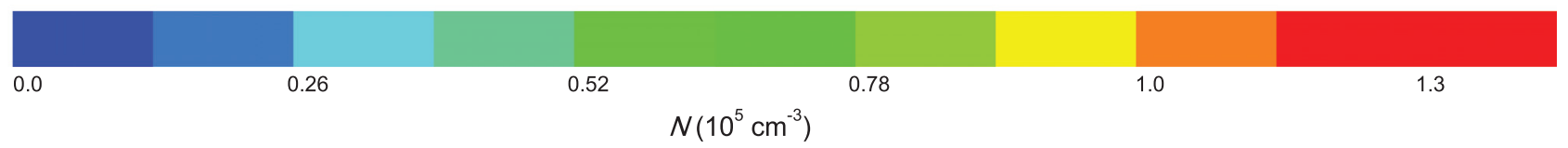

FIG. 6. Spatial distribution of the $\operatorname{Ar} I\left[3 s^{2} 3 p^{5}\left({ }^{2} P_{1 / 2}^{0}\right) 4 p, J=1\right]$ population density using filtered back projection with Shepp-Logan filter obtained at distance (a) $3 \mathrm{~cm}$ from the cavity, (b) $4.5 \mathrm{~cm}$ from the cavity, and (c) $8 \mathrm{~cm}$ from the cavity. 
The lack of cylindrical symmetry in the population distributions emerges from our ability to measure a limited range of angles $\left(48^{\circ}-168^{\circ}\right)$. The missing parts correspond to the area that was not covered in the experiment. It is, however, obvious from the plots that the excited species are dominantly populated at the rim of the plasmoid. This observation indicates that the plasmoid is sustained by a surface wave. Ongoing experiments on the plasmoid will help us determine the temperature and concentration profiles throughout the plasmoid to fully characterize it and reveal the nature of the surface wave that sustains it.

\section{B. Electron excitation temperature}

The electron excitation temperatures were determined employing the Boltzmann plot method. This method assumes that the spectral irradiances $\left(I_{k i}\right)$ of different Ar excited state spectral lines having the same lower energy and different threshold excitation energies $\left(E_{k}\right)$ follow a Botzmann distribution

$$
I_{k i}=\frac{g_{k} A_{k i}}{\lambda_{k i}} e^{-E_{k} / k_{B} T_{e x c}}
$$

where $g_{k}$ is the statistical weight of the upper level $k, A_{k i}$ is the transition probability, and $\lambda_{k i}$ is the wavelength. The electron excitation temperature relates to the slope of the $\log \left(I_{k i} \lambda_{k i} / g_{k} A_{k i}\right)$ versus $E_{k}$ plot. We were looking into transitions to the Ar I $3 s^{2} 3 p^{5}\left({ }^{2} P_{3 / 2}^{0}\right) 4 s, J=2$ lower level. The upper energy levels, wavelengths, and corresponding constants used for determining the excitation temperature are presented in Table I.

Equation (13) incorporates a premise that discharge satisfies the LTE or, at least, is close to it. The validity criteria for LTE were established by Griem. ${ }^{11}$

$$
N_{e}=7 \times 10^{18} \frac{z^{7}}{n^{17 / 2}}\left(\frac{k T_{e}}{z^{2} E_{H}}\right) \mathrm{cm}^{-3},
$$

where $N_{e}$ is the electron density, $z=1$ for neutral emitters, $n$ is a principal quantum number of the upper level $(n=4$ for $\operatorname{argon}), E_{H}$ is the ionization energy of hydrogen, and $T_{e}$ is the electron temperature.

Based on evaluations ${ }^{12}$ of $T_{e}$, we can assume that $k T_{e}=0.5 \mathrm{eV}$, meaning that an electron density of $N_{e}$ $\approx 10^{13} \mathrm{~cm}^{-3}$ is required to fulfill LTE. This condition is satisfied in our case ${ }^{12}$ that justifies the Boltzmann plot approach in calculating $T_{\text {exc }}$.

TABLE I. Ar I spectral lines used for $T_{\text {exc }}$ determination.

\begin{tabular}{lccccc}
\hline \hline Upper level & $J$ & $\lambda(\mathrm{nm})$ & $g_{k}$ & $A_{k i}\left(10^{6} \mathrm{~s}^{-1}\right)$ & $E_{k}\left(\mathrm{~cm}^{-1}\right)$ \\
\hline $3 s^{2} 3 p^{5}\left({ }^{2} P_{3 / 2}^{0}\right) 5 p$ & 2 & 415.9 & 5 & 1.400 & 117183 \\
$3 s^{2} 3 p^{5}\left({ }^{2} P_{3 / 2}^{0}\right) 5 p$ & 3 & 420.1 & 7 & 0.967 & 116942 \\
$3 s^{2} 3 p^{5}\left({ }^{2} P_{1 / 2}^{0}\right) 4 p$ & 2 & 706.8 & 5 & 3.860 & 107289 \\
$3 s^{2} 3 p^{5}\left({ }^{2} P_{1 / 2}^{0}\right) 4 p$ & 1 & 714.7 & 3 & 0.625 & 107131 \\
$3 s^{2} 3 p^{5}\left({ }^{2} P_{3 / 2}^{0}\right) 4 p$ & 3 & 811.5 & 7 & 33.10 & 105462 \\
\hline \hline
\end{tabular}

a) $3 \mathrm{~cm}$ from the cavity

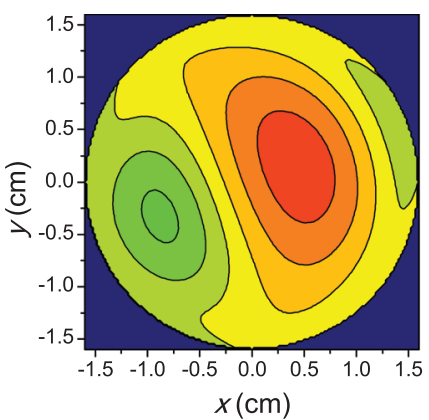

b) $8 \mathrm{~cm}$ from the cavity

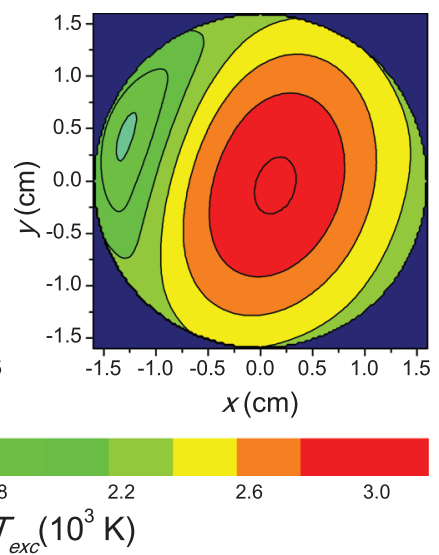

FIG. 7. Spatial distributions of argon excitation temperatures obtained at distances (a) $3 \mathrm{~cm}$ from the cavity and (b) $8 \mathrm{~cm}$ from the cavity.

We obtained the distributions of excitation temperatures at two positions along the plasmoid, $3 \mathrm{~cm}$ and $8 \mathrm{~cm}$ from the cavity corresponding to the beginning and ending of the plasmoid, respectively. Results are presented in Fig. 7. The maximal excitation temperature obtained was $T_{e x c}=2820 \mathrm{~K}$ $\times 3 \mathrm{~cm}$ from the cavity and $T_{e x c}=3030 \mathrm{~K} \times 8 \mathrm{~cm}$ from the cavity. This is in good agreement with previously published data. $^{12}$

Note that the excitation temperature maximum tends to be in contrast to the population distribution. Furthermore, the temperature distributions exhibit rotational behavior. Both effects reflect the influence of the supersonic flow. Relative populations of the two excited levels tend to follow the dynamics and structure of the flow, which in turn is affected by the surface wave discharge. The phenomenon is nonlinear, and more detailed spectroscopy is needed to clarify the flow dynamics.

\section{CONCLUSION}

A 2D plasma tomography model has been applied for obtaining the spatial distributions of the argon excited levels in supersonic flowing MW discharge. The technique is based on the inverse Radon transform formula for a cylindrical cavity geometry. Optical emission spectroscopy measurements were performed by employing an automated measurement system. The AMS was built to streamline the measurements and to increase the overall precision. The system controls the angle and distance within sub-degree and sub-millimeter angle and distance precision, respectively. We obtained the spatial distributions of two neutral Ar excited states $\left[3 s^{2} 3 p^{5}\left({ }^{2} P_{1 / 2}^{0}\right) 4 p, J=2\right]$ and $\left[3 s^{2} 3 p^{5}\left({ }^{2} P_{1 / 2}^{0}\right) 4 p, J=1\right]$ at different positions along the plasmoid. The $2 \mathrm{D}$ distributions of the electron excitation temperatures were determined from the Boltzmann plot method, assuming partial local thermodynamic equilibrium. The results indicate that the discharge is sustained by a surface wave, but it is also highly affected by the supersonic flow. While the population peaks in the vicinity of the tube walls, the excitation temperature has a broad maximum close to the discharge axes. The locations of temperature maxima vary with the downstream longitudinal position. 


\section{ACKNOWLEDGMENTS}

This work is supported by the Office of the High Energy Physics, Office of Science, Department of Energy under Grant No. DE-SC0007879. Thomas Jefferson National Accelerator Facility supports M. Nikolić through fellowship.

${ }^{1}$ V. K. Liao, M. T. C. Fang, J. D. Yan, and A. I. Al-Shammaa, J. Phys. D: Appl. Phys. 36, 2774 (2003).

${ }^{2}$ M. Baeva, A. Bosel, J. Ehlbeck, and D. Loffhagen, Phys. Rev. E 85, 056404 (2012).
${ }^{3}$ R. Alvarez, A. Rodero, and M. C. Quintero, Spectrochim. Acta, Part B 57, 1665 (2002).

${ }^{4}$ J. Radon, IEEE Trans. Med. Imaging 5, 170 (1986) (Translated by P. C. Parks from the original German text).

${ }^{5}$ M. P. Freeman and S. Katz, J. Opt. Soc. Am. 53, 1172 (1963).

${ }^{6}$ K. G. Lapworth and L. A. Allnutt, J. Phys. E: J. Sci. Instrum. 10, 727 (1977).

${ }^{7}$ T. Makabe and Z. L. Petrović, Appl. Surf. Sci. 192, 88-114 (2002).

${ }^{8}$ A. Samolov, Ph.D. dissertation, Old Dominion University, 2012.

${ }^{9}$ M. J. Lighthill, Introduction to Fourier Analysis and Generalised Functions (Cambridge University Press, Cambridge, Great Britain, 1959).

${ }^{10}$ L. A. Shepp and B. F. Logan, IEEE Trans. Nucl. Sci. 21, 228 (1974).

${ }^{11}$ H. R. Griem, Plasma Spectroscopy (McGraw-Hill, New York, 1964).

${ }^{12}$ D. J. Drake, S. Popović, and L. Vušković, J. Appl. Phys. 104, 063305 (2008). 